\title{
The MoEDAL experiment at the LHC
}

\author{
James Pinfold ${ }^{\mathrm{a}}$ \\ Physics Department, University of Alberta, Edmonton, Alberta, Canada
}

\begin{abstract}
MoEDAL is a pioneering experiment designed to search for highly ionising messengers of new physics such as magnetic monopoles or massive (pseudo-)stable charged particles, that are predicted to exist in a plethora of models beyond the Standard Model. Its ground-breaking physics program defines a number of scenarios that yield potentially revolutionary insights into such foundational questions as: are there extra dimensions or new symmetries; what is the mechanism for the generation of mass; does magnetic charge exist; what is the nature of dark matter; and, how did the big-bang develop at the earliest times. MoEDAL's purpose is to meet such far-reaching challenges at the frontier of the field. In conclusion we will briefly report on current results; discuss plans to instal a new detector designed to search for very long-lived neutral particles as well as mini-charged particles; and, briefly delineate plans for an astroparticle extension of MoEDAL called Cosmic-MoEDAL.
\end{abstract}

\section{Introduction}

MoEDAL (Monopole and Exotics Detector at the LHC) [1-3], the 7th experiment at the Large Hadron Collider (LHC) [4], was approved by the CERN Research Board in 2010. It is designed to search for manifestations of new physics through highly-ionising particles in a manner complementary to ATLAS and CMS [5]. The most important motivation for the MoEDAL experiment is to pursue the quest for magnetic monopoles and dyons at LHC energies. Nonetheless the experiment is also designed to search for any massive, stable or long-lived, slow-moving particles $[6,7]$ with single or multiple electric charges arising in many scenarios of physics beyond the Standard Model (SM). A selection of the physics goals and their relevance to the MoEDAL experiment are described here and elsewhere [8]. For an extended and detailed account of the MoEDAL discovery potential, the reader is referred to the recently published MoEDAL Physics Review [9].

The structure of this paper is as follows. Section 2 provides a brief description of the MoEDAL detector. The physics reach of MoEDAL is discussed in Sect. 3, whilst Sect. 4 is dedicated to a discussion of recent results. Section 5 deals with the proposal to instal the MoEDAL detector for Penetrating Particles (MAPP) and a possible astroparticle extension of the MoEDAL-LHC project i.e. Cosmic-MoEDAL.

\section{The MoEDAL detector}

The MoEDAL detector [2] is deployed around the intersection region at Point 8 of the $\mathrm{LHC}$ in the $\mathrm{LHCb}$ experiment Vertex Locator (VELO) [10] cavern. A threedimensional depiction of the MoEDAL experiment is presented in Fig. 1. It is a unique and largely passive LHC detector comprised of three sub-detector systems,

a e-mail: jpinfold@ualberta.ca with a fourth in the planning stage. MoEDAL bypasses the experimental difficulties encountered by the main LHC experiments in the detection of Highly Ionising Particle (HIP) messengers of new physics by using a passive plastic NTD technique to detect the ionisation trail of HIPs as well as a novel trapping array - called the MMT (Magnetic Monopole Trapper) for detecting HIPs that slow down and stop within its sensitive volume. Neither of these detector systems requires trigger or read-out electronics.

As the MoEDAL NTD stacks are less than 1-cm-thick there is little chance that a HIP will be absorbed. Also, NTDs provide a tried-and-tested and cost-effective method to accurately measure the track of a HIP and its effective charge. Importantly, MoEDAL's exposed HIP films will be directly calibrated in a heavy-ion beam. MoEDAL's roughly one tonne of MMT detectors insures that a small but significant fraction of the HIPs produced will be trapped for further study in the laboratory. MoEDAL's ability to retain a permanent record, and even capture new particles for further study - will make it an invaluable asset in the elucidation of any Terascale BSM scenario covered by its extensive physics repertoire. There are no SM particles that can produce such distinct signatures thus, even the detection in MoEDAL of few HIP particle messengers of new physics would herald a discovery. The only 'real-time' sub-detector system is the TimePix2 pixel array that will be used to monitor low-energy highly ionising beam-related backgrounds.

\subsection{The NTD detector sub-system}

The Low Threshold (LT-NTD) array, part of the NTD subdetector system, is the largest array of NTD detectors ever deployed at an accelerator. The NTD plastics employed are polyallyl-diglygol-carbonate (PADC) commonly known as CR39 Ra transparent rigid plastic and the polycarbonate Makrofol $\AA$. Each of the roughly $300\left(25 \times 25 \mathrm{~cm}^{2}\right)$ LT-NTD stacks is comprised of three sheets of CR39 R and three of Makrofol as shown in Fig. 2. The CR39 layers

(C) The Authors, published by EDP Sciences. This is an Open Access article distributed under the terms of the Creative Commons Attribution License 4.0 (http://creativecommons.org/licenses/by/4.0/). 


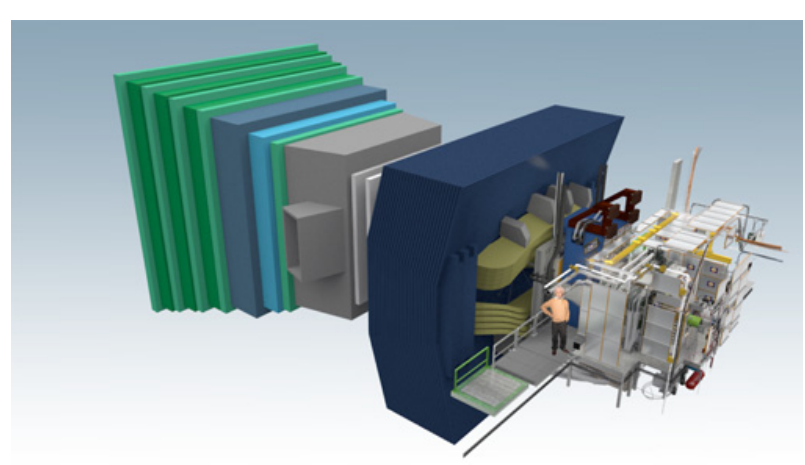

Figure 1. A schematic view of the MoEDAL detector.

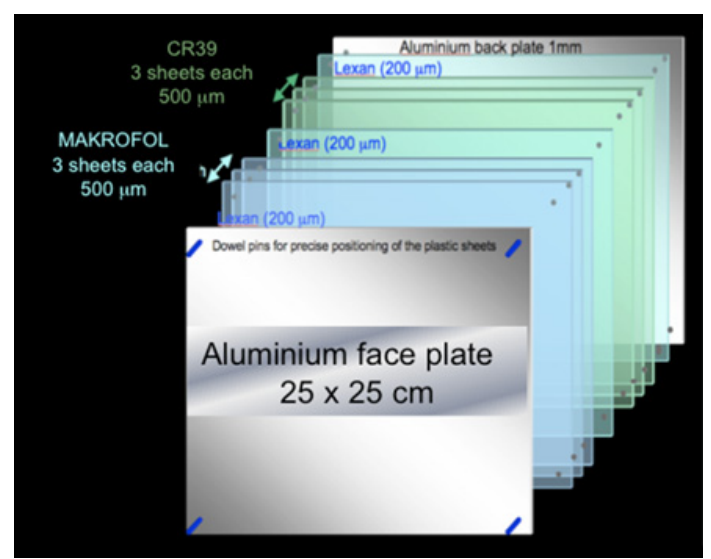

Figure 2. A MoEDAL stack comprised of CR39 and Markrofol NTD detectors.

in the LT-NTD array can detect particles with ionisation equivalent to five normal particles with charge resolution, better than $0.2 \mathrm{e}$, where $\mathrm{e}$ is the charge of an electron.

The TDR NTD array has been enhanced by the high charge catcher (HCC) sub-detector - with threshold Z/ $\beta$ of approximately 50 - comprised of stacks of three Makrofol plastic sheets in an Al foil envelope. These lightweight low-mass detector stacks can be deployed in previously inaccessible areas on and around LHCb's VELO (Vertex Locator) detector increasing the geometrical acceptance for magnetic monopoles to $\sim 60 \%$. Importantly, a plane of roughly $4 \mathrm{~m}^{2}$ of $\mathrm{HCC}$ detectors - termed 'the shower curtain' - has been placed in the forward acceptance of the LHCb detector between the LHCb's RICH (Ring Imaging Cherenkov detector) and its first station of tracking detectors (TT) where the material budget is at a minimum. Although NTD technology was used in the past to search for monopoles [11], this is the first time at an accelerator that such a large area array of NTDs has been used to search for a wide range of magnetically and electrically charged HIPs.

The passage of a highly-ionising particle through the plastic detector is marked by an invisible damage zone along the trajectory. The damage zone is revealed as a cone-shaped etch-pit when the plastic detector is etched using a hot sodium hydroxide solution. Then the sheets of plastics are scanned looking for aligned etch pits in multiple sheets using "intelligent" computer controlled optical scanning microscopes. A HIP with ionising power greater than or equal to 5 times that of a relativistic charged SM particle will leave a characteristic set of at least 6 collinear etch pits in the 3 CR39 sheets in each

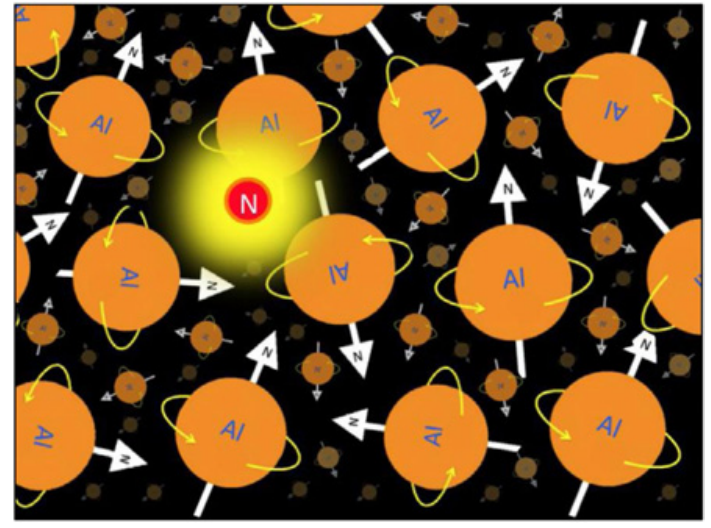

Figure 3. A monopole captured within an aluminium trapping element of the MoEDAL Trapping detector.

NTD stack. Magnetic monopoles, each with an ionising power that is thousands of times that of a SM particle will leave an unprecedented trail of 12 etch pits in a MoEDAL stack with 6 NTD sheets. These aligned etch pits, of size typically in the range $20-50$ microns, accurately define a track that points towards to Interaction Point (IP). There is no known Standard Model background to this signal.

\subsection{The trapping detector sub-system}

The MMT is the newest sub-detector system to be added to the MoEDAL detector. The MMT detector consists of roughly 1 tonne of aluminium $(\mathrm{Al})$ paramagnetic volumes placed at three points around the intersection point, IP8, that MoEDAL shares with the $\mathrm{LHCb}$ experiment. Al has an enhanced capability to trap monopoles due to its anomalously large nuclear magnetic moment. A fraction of the massive HIPs created will stop and be captured in the MMT detector as illustrated in Fig. 3. MoEDAL is the first experiment to use purpose-made trapping volumes to capture magnetic and electrically charged particles. The exposed monopole trapping volumes are monitored at the ETH Zurich SQUID facility for the presence of captured monopoles, a schematic description of the facility is given in Fig. 4. The use of SQUIDs to detect trapped magnetic charge has also been thoroughly tested in particle and astroparticle experiments where the search has been performed on 'found' or alternate use objects (such as beam-pipes) on a 'one-off' basis.

The signal for a magnetic monopole in the monopole trapping detectors at the ETH facility would be a sustained current - resulting from the passage of a monopole through the SQUID detector. Test solenoids are used to calibrate the response of the SQUID to a trapped monopole. Using test solenoids we found that the SQUID can detect magnetic charges as small as $0.1 \mathrm{~g}$. After the SQUID scan has been performed it is envisaged that the trapping volumes will be sent to SNOLAB $-2 \mathrm{~km}$ underground - to be monitored for the decays of very long-lived electrically charged particles $\left(\tau>10^{7} \mathrm{~s}\right)$. The MoEDAL search for very long-lived particles using a dedicated detector deployed deep underground has some advantages over searches carried out with the LHC GPEs. For example, MoEDAL has no trigger requirement and thus can perform relatively model-independent studies. Using long test solenoids to mimic a monopole we have 


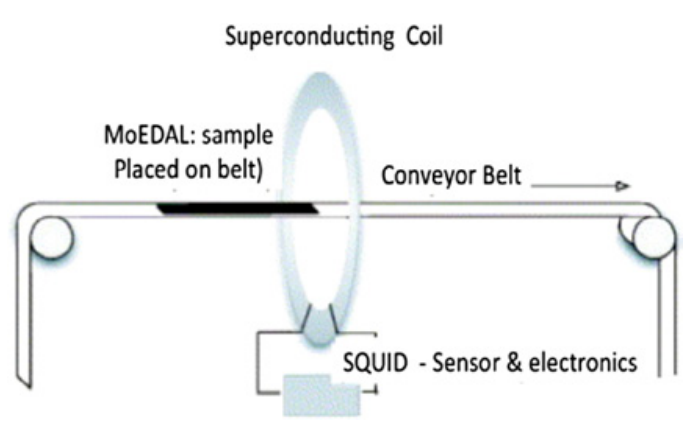

Figure 4. A schematic depiction of the use of a SQUID magnetometer to detect the presence of a trapped magnetic charge.

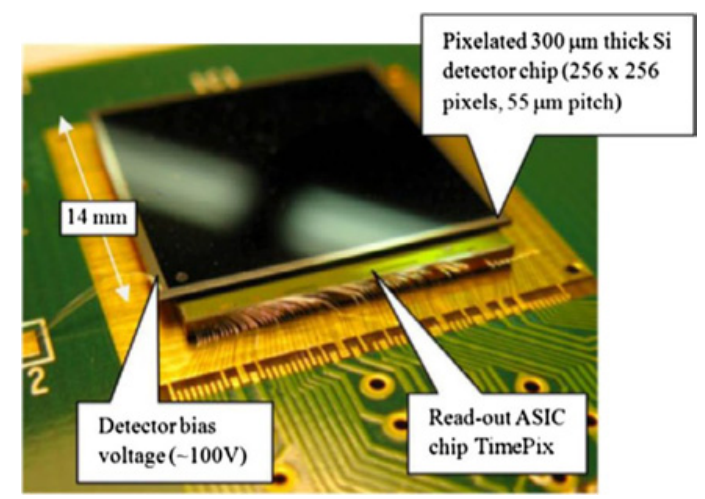

Figure 5. A photograph of a TimePix pixel device.

determined that the SQUID can detect trapped monopole with magnetic charge as small as 0.1 of a Dirac charge $\left(0.1 g_{D}\right)$.

\subsection{The TimePix radiation monitoring system}

An array of six TimePix 2 pixel detectors are used to monitor the low energy highly-ionizing beam related backgrounds. Each pixel of the TimePix chip contains a preamplifier to enhance the signal, a discriminator to severely reduce electronic noise and a 4-bit DAC for threshold adjustment, and a 14-bit counter. MoEDAL uses the TimePix device's 'Time-over-Threshold' (ADC) and 'Time of Arrival' modes so that each pixel can supply an energy measurement. A photograph of a TimePix pixel chip is shown in Fig. 5. The TimePix detector is capable of providing a colour image of complete spallation events in its 300 micron thick silicon sensitive volume - with energy encoded in the colour.The TimePix sub-detector is read out via the web.

\section{The physics reach of MoEDAL}

As discussed above, the standard general-purpose Collider detectors at the LHC are not designed or optimised to detect massive slow-moving HIPs or mQPs. MoEDAL's new light on the high-energy frontier is provided by the use of massive HIPs or mQPs - for which there are no SM counterparts - as direct probes of pioneering new physics at the Terascale. Such an approach requires a new state-ofthe-art in the quest for massive HIP messengers of beyond the SM physics that is provided by the custom-designed MoEDAL experiment.

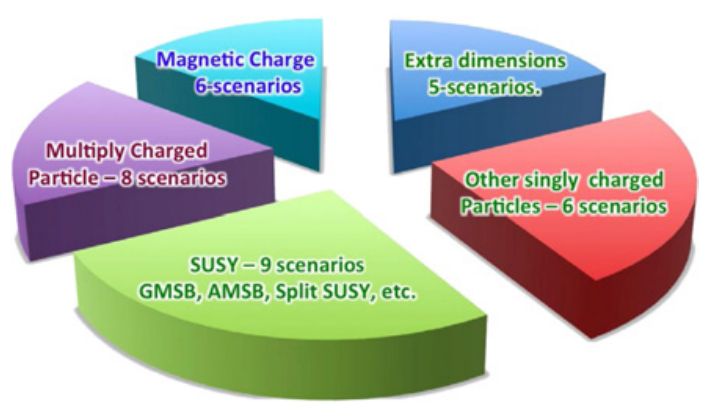

Figure 6. MoEDAL Physics Programme defined in Ref. [9] broken down by topic. We have identified 34 different existing possible discovery scenarios.

MoEDAL's physics programme [9] covers many fundamentally important BSM scenarios allowing MoEDAL to significantly expand the LHC's discovery horizon in a complementary way, as illustrated in the pie chart shown in Fig. 6. MoEDAL's Physics Coordinator and Theory Board oversee the physics programme in order to ensure that the physics objectives are maximised and met. The MoEDAL physics programme addresses fundamental questions such as: does magnetic charge exist, are there extra dimensions; are there new symmetries of nature; and, what is the nature of dark matter.

One of the main objectives for MoEDAL is the stateof-the-art search for the magnetic monopole, just as the search for the Higgs was the prime motivation for the LHC and its GPEs. The work of our Theory Board has renewed interest in the LHC search for a Terascale EW monopole $[9,12]$ arising from the SM. If discovered, this would be the first topological particle to be observed with the utmost consequences for our understanding of the Universe. But MoEDAL is designed to do much more, as indicated in Fig. 6 by probing BSM physics by searching for anomalously electrically charged particles.

\section{Recent MoEDAL results}

The first MoEDAL results utilized a $160 \mathrm{~kg}$ of prototype MoEDAL trapping detector exposed to $8-\mathrm{TeV}$ protonproton collisions at the LHC, for an integrated luminosity of $0.75 \mathrm{fb}^{-1}$ during LHC's Run I. No magnetic charge exceeding $0.5 \mathrm{gD}$ was detected in any of the exposed samples, allowing limits to be placed on monopole production in the mass range $100 \mathrm{GeV} \leq M_{\text {monopole }} \leq$ $3500 \mathrm{GeV}$ [13]. Model-independent cross-section limits have been presented in fiducial regions of monopole energy and direction for $1 \mathrm{~g}_{D} \leq|g| \leq 6 \mathrm{~g}_{D}$, and modeldependent cross-section limits are obtained for Drell-Yan (DY) pair production of spin-1/2 and spin-0 monopoles for $1 \mathrm{~g}_{D} \leq|g| \leq 4 \mathrm{~g}_{D}$. Under the assumption of Drell-Yan cross sections, mass limits are derived for $j g j=2 \mathrm{~g}_{D}$ and $|g|=3 \mathrm{~g}_{D}$ for the first time at the LHC, surpassing the previous result from the ATLAS Collaboration [14,15] which placed limits only for monopoles with magnetic charge $|g|=1 \mathrm{~g}_{D}$.

The first search for magnetic monopole production in $13 \mathrm{TeV}$ proton-proton collisions during LHC's Run-2 using the trapping technique have extended the previous results with $8 \mathrm{TeV}$ data during LHC's Run-1. In this case a total of $222 \mathrm{~kg}$ of MoEDAL trapping detector samples was exposed in the forward region and 


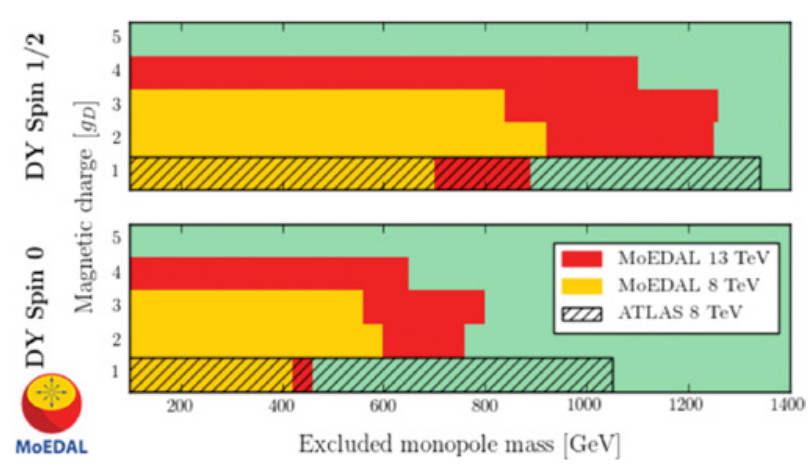

Figure 7. Monopole lower mass limits (95\% confidence level) in models of spin-1/2 and spin-0 DY pair production in LHC pp collisions for monopole charges $|g|$ up to $4_{D}$. These limits are based upon cross sections computed at leading order. These cross sections are only indicative since the monopole coupling to the photon is too large to allow for perturbative calculations. Previous results obtained in $8 \mathrm{TeV}$ collisions are from Ref. [13] (MoEDAL prototype trapping detector) and Ref. $[14,15]$ (ATLAS).

analysed by searching for induced persistent currents after passage through a superconducting magnetometer. Magnetic charges exceeding half the Dirac charge are excluded in all samples and limits are placed for the first time on the production of magnetic monopoles in $13 \mathrm{TeV}$ pp collisions. This search probes mass ranges previously inaccessible to collider experiments for up to five times the Dirac charge. A summary of the limits obtained by both the above analyses are summarized in Fig. 7.

\section{Future MoEDAL developments}

A key aspect of the MoEDAL experiment is that it is sensitive to massive slow-moving and very HIPs, messengers of new physics for which the standard LHC GPE detectors are not optimised. MoEDAL is preparing a proposal to add a new sub-detector that is sensitive to particles with charge as small as a thousandth that of the electron - a mini-charged particle (mQP). The main LHC detectors are essentially blind to such particles. This addition to its detector system is consistent with MoEDAL's ethos of extending the physics reach of the LHC by searching for anomalously charged messengers of new physics in a way that is complementary to the existing capability provided by the main LHC detectors.

MoEDAL is proposing to deploy the MAPP (MoEDAL apparatus for detecting penetrating particles) in a tunnel shielded by some $30 \mathrm{~m}$ to $50 \mathrm{~m}$ of rock and concrete from the interaction point (IP8), as shown in Fig. 8. The purpose of the detector is to search for particles with fractional charge as small as one-thousandth the charge of an electron. This detector would also be sensitive to neutral particles from new physics scenarios via their interaction or decay in flight within the volume of the detector. The isolation of the detector means that the huge background from SM processes in the main detectors is largely absent.

The first apparatus specifically designed to detect mini-charged particles was the SLAC (Stanford Linear Accelerator Centre) 'beam dump' type detector, comprised of scintillator bars read out by photomultiplier tubes [16]. MoEDAL's new detector, shown in Fig. 9, and another apparatus proposed for deployment near to the CMS detector [17] also designed to search for minicharged

\section{IP8 /UX85 - MoEDAL Millicharged Part.Det.}

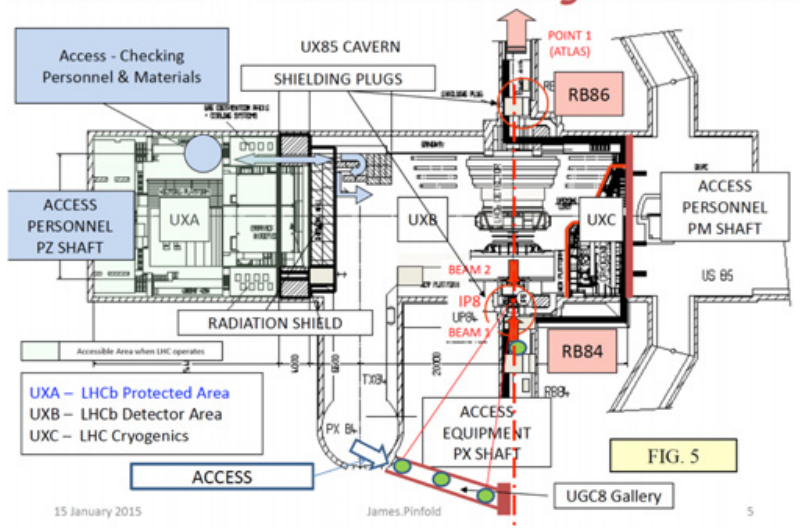

Figure 8. MoEDAL is preparing a proposal to install a minicharged particle detector positioned in the forward region of IP8 some $30 \mathrm{~m}$ from the $\mathrm{LHCb} / \mathrm{MoEDAL}$ intersection point (IP8).

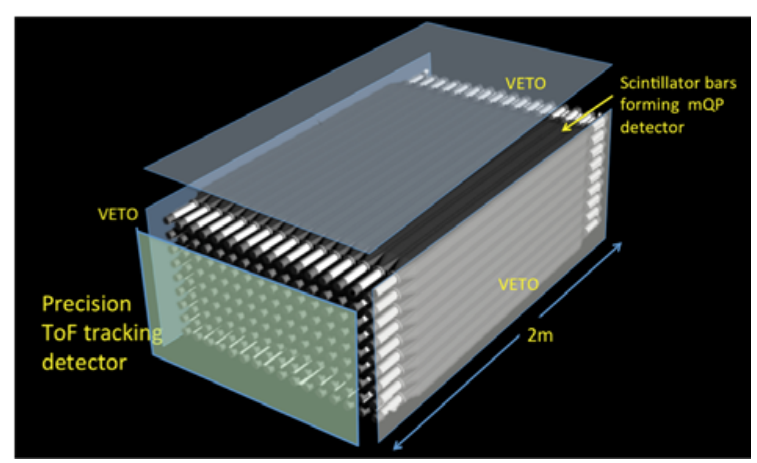

Figure 9. A depiction of a MAPP detector subunit. The final detector could contain up to four such units.

particles, both have a design that harks back to the original SLAC detector. In order to reduce backgrounds from natural radiation the photomultiplier tubes and scintillator detectors of the MoEDAL apparatus will be constructed from materials with low natural backgrounds currently utilised in the astroparticle physics arena. Its calibration system utilises neutral density filters to reduce the received light of high incident muons that manage to penetrate to the sheltered detector from the interaction point, in order to mimic the much lower light levels expected from particles with fractional charges.

\subsection{Cosmic-MoEDAL}

The MoEDAL collaboration is preparing an astroparticle extension to the MoEDAL-LHC experiment that will enable the search, for example, for magnetic monopoles to be extended from $\mathrm{TeV}$ scale at the LHC up to the Grand Unification (GUT) scale. In addition we propose to use the same detector technology for 'Cosmic-MoEDAL' as we use for MoEDAL-LHC. SLIM was the first experiment to use such an approach to extend the search for cosmic monopoles with masses from the GUT scale well below the GUT scale, with a high sensitivity. SLIM was necessarily deployed at high altitude at the Mt Chacaltaya lab. in Bolivia with an elevation of 5,400 m. However, SLIM's modest size $\left(400 \mathrm{~m}^{2}\right)$ precluded it from the search for a flux of cosmic monopoles below the Parker Bound (an upper bound on the density of magnetic monopoles that 


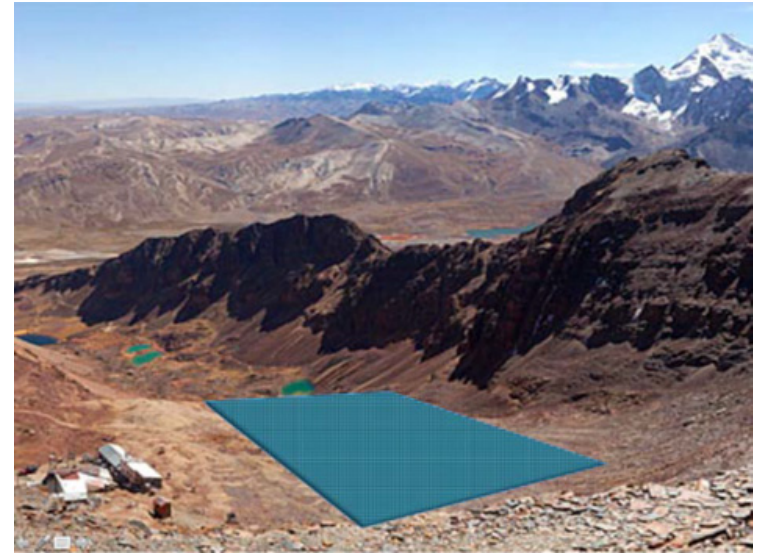

Figure 10. Artist's impression of the Cosmic-MoEDAL array deployed at an altitude of $5 \mathrm{~km}$ on Mt Chacaltaya.

is obtained from arguments based on the existence of a galactic magnetic field).

Cosmic-MoEDAL is proposed as a 50,000-100,000 $\mathrm{m}^{2}$ of plastic NTDs (CR39) deployed at high altitude. Such an array would be able to take the search for cosmic monopoles with velocities $\beta \gtrsim 0.1$ from the TeV scale to the GUT scale for monopole fluxes well below the Parker Bound. Possible sites for Cosmic MoEDAL include: Chacaltaya $(5 \mathrm{~km})$ and Tenerife-Tiede $(3 \mathrm{~km})$. An artists impression of Cosmic-MoEDAL on Mt Chacaltaya is shown in Fig. 10.

\section{Summary and conclusion}

In 2015, the LHC restarted operations at the unparalleled energy of $6.5 \mathrm{TeV}$ beam energy and the enormous collision rate of around a billion collisions per second. The LHC has been compared to a time machine enabling us to recreate the conditions that occurred when the Universe was only roughly 100 picoseconds old, providing an unprecedented laboratory for the study of the cosmology of the nascent Universe at the earliest times. In parallel, non-accelerator experiments are also exploring the Tera-universe via highenergy astrophysics.

The first deployment of the MoEDAL detector employing largely passive detector systems, tuned to the prospect of discovery physics, was at Point 8 on the LHC ring, in the Winter of 2014. As we have seen the novel MoEDAL detector, has a dual nature. First, it acts like a giant camera, comprised of NTDs - analysed offline by ultra fast scanning microscopes - sensitive only to new physics. Second, it is uniquely able to trap the particle messengers of physics beyond the SM for further study. MoEDAL's radiation environment is monitored by a stateof-the-art real-time TimePix pixel detector array. A new MoEDAL sub-detector, MAPP, sensitive to mini-charged particles and long-lived neutral particles, is being studied.

The first MoEDAL results using only a small part of their overall detector system have provided the world's best limits on multiply charge monopoles. In 2017 and beyond we expect to exploit MoEDAL's full detector repertoire to provide further world class limits on highly ionizing magnetically and electrically charged particles from a number of well predicated theoretical scenarios. In addition, MoEDAL has an aggressive plan to extend the physics reach of MoEDAL LHC with the MAPP detector and a possible astroparticle extension to MoEDAL, called Cosmic MoEDAL, that will be capable of taking the search for, for example, magnetic monopoles up to the GUT scale.

July 2012 the ATLAS and CMS experiments operating at the LHC announced the discovery of the Higgs boson. But, the LHC is only just getting started. Many fundamental questions still remain to be answered. Are there new symmetries of nature? Are there extra spatial dimensions? Is there a deeper substructure? Does magnetic charge exist? Why is gravity so weak compared to the other fundamental forces? What is the nature of dark matter? What was the physics of the earliest era of the universe? The list goes on. MoEDAL is designed to provide insights into such fundamental questions.

\section{References}

[1] For general information on the MoEDAL experiment, see: http://moedal.web.cern.ch/

[2] MoEDAL Collaboration, Technical Design Report of the MoEDAL Experiment CERN Preprint CERNLHC-2009-006, MoEDAL-TDR-1.1 (2009), and references therein

[3] M. Fairbairn and J. L. Pinfold, published online by Contemporary Physics, http://www.tandf online.com/doi/full/10.1080/00107514. 2016.1222649

[4] L. Evans and P. Bryant, JINST 3, S08001 (2008)

[5] A. De Roeck, A. Katre, P. Mermod, D. Milstead and T. Sloan, Eur. Phys. J. C 72, 1985 (2012)

[6] M. Fairbairn, A. C. Kraan, D. A. Milstead, T. Sjostrand, P. Z. Skands and T. Sloan, Phys. Rept. 438, 1 (2007)

[7] S. Burdin, M. Fairbairn, P. Mermod, D. Milstead, J. Pinfold, T. Sloan andW. Taylor, Phys. Rept. 582, 1 (2015)

[8] V. A. Mitsou [MoEDAL Collaboration], EPJ Web Conf. 95 (2015) 04042

[9] B. Acharya et al. [MoEDAL Collaboration], Int. J. Mod. Phys. A 29, 1430050 (2014)

[10] A. A. Alves, Jr. et al. [LHCb Collaboration], JINST 3, S08005 (2008)

[11] K.A. Olive, K. Agashe, C. Amsler, M. Antonelli, J.-F. Arguin, D.M. Asner, et al., Particle Data Group, Magnetic monopole searches, Chin. Phys. C 38, 090001 (2014)

[12] Y.M. Cho and D. Maison, Phys. Lett. B 391 (1997)

[13] B. Acharya et al. [MoEDAL Collaboration], JHEP 1608, 067 (2016)

[14] G. Aad et al. [ATLAS Collaboration], Phys. Rev. Lett. 109, 261803 (2012)

[15] G. Aad et al. [ATLAS Collaboration], Phys. Rev. D 93(5), 052009 (2016)

[16] A.A. Prinz, et al., Phys. Rev. Lett. 81, 1175 (1998)

[17] A. Haas, C.S. Hill, E. Izaguirre, and I. Yavin, Looking for millicharged particles with a new experiment at the LHC, Phys. Lett. B 746, 117 (2015) 